Authors have nothing to disclose with regard to commercial support.

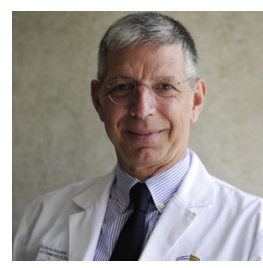

\section{PREDICTABILITY OF ACUTE AORTIC DISSECTION. A DREAM COME TRUE?}

To the Editor:

We read with great interest and pleasure the article by Zafar and colleagues, ${ }^{1}$ as well as the accompanying commentary. The Elefteriades group is probably the only one in the world able to correct the aortic size index (ASI) for the very reason they were able to develop it: their large database of patients with thoracic aortic ascending aneurysms with good followup. In other words, the group has both cases (patients with dissections and ruptures) and controls (patients with thoracic ascending aortic aneurysms under the threshold of surgical indication and therefore under follow-up). The most interesting and reasonable correction was the abolition of the use of body surface area (BSA) and thus of body weight from the equation, a kind of overweight or obesity paradox of the old ASI: the more overweight or obese the patient, the lower the potential risk of rupture or dissection. Looking at our own results, which come from an interesting epidemiologic constellation (108 patients from a single referral center in a geographically circumscribed region of Switzerland with approximately 350,000 inhabitants), the prevalence of overweight or obese patients among those older than 17 years presenting with acute aortic type A dissection (AADA) is $65 \%$, statistically significantly higher than in the reference population. Overweight or obesity thus is an independent risk factor, confirming the ASI overweight paradox (presented in June 2016 at the Annual Meeting of the Swiss Societies of Cardiology and Cardiovascular Surgery). Completely abolishing the use of BSA (and thus body weight) or adjusting for a normal BSA (and body weight) would have shifted $26 \%$ of our patients from a low to a moderate risk of rupture or dissection (had these patients had thoracic aortic aneurysms diagnosed before their inaugural event, the AADA). The second correction of the ASI is the independent lowering of the threshold of surgical indication. Whereas the old threshold to moderate risk was $29 \mathrm{~mm} / \mathrm{m}$ of height (calculation without BSA and with height only, as with the new aortic height index), analyzing the new recommendations, the transition to moderate risk of rupture or dissection is now at approximately $24 \mathrm{~mm} / \mathrm{m}$, a significant difference. ${ }^{1}$ Applying a posteriori all new recommendations to our group of 100 patients, $91 \%$ presented with AADA, which would have been theoretically preventable!
We congratulate the Elefteriades group for this milestone contribution, which paves the way for a more aggressive attitude toward proactive noninvasive screening of thoracic ascending aortic aneurysms and prophylactic surgery. After all, with aggressive lowering of the risk factors (mainly arterial hypertension), generous screening, especially in individuals with positive family history for aortic aneurysms, and aggressive prophylactic replacement of the ascending aorta, AADA could indeed be preventable. Bundled, these measures could prove both lifesaving and cost-effective, considering the high mortality and the secondary acute and chronic costs associated with AADA.

Stefanos Demertzis, $M D$
Susanna Grego, $M D$
Cardiocentro Ticino
University of Bern
University of Svizzera Italiana
Lugano, Switzerland

\section{Reference}

1. Zafar MA, Li Y, Rizzo JA, Charilaou P, Saeyeldin A, Velasquez CA, et al. Height alone, rather than body surface area, suffices for risk estimation in ascending aortic aneurysm. J Thorac Cardiovasc Surg. 2018;155:1938-50.

https://doi.org/10.1016/j.jtcvs.2019.01.026

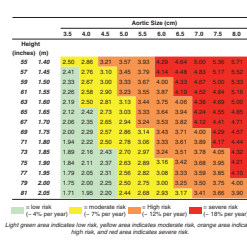

\section{PREDICTABILITY OF ACUTE AORTIC DISSECTION: A DREAM IN EVOLUTION \\ Reply to the Editor:}

We thank Demertzis and Grego ${ }^{1}$ for their very kind commentary, "Predictability of Acute Aortic Dissection: A Dream Come True?" regarding our recent article entitled "Height Alone, Rather Than Body Surface Area, Suffices for Risk Estimation in Ascending Aortic Aneurysm." ${ }^{2}$ We are thrilled that Demertzis and Grego ${ }^{1}$ find our latest predictive tool, which is based only on height, meaningful and useful.

Our earlier tools, which followed common clinical practice in many arenas, calculated the significance of a specific aortic size by weighing against body surface area (BSA). As Demertzis and Grego ${ }^{1}$ so clearly point out, an obese patient would have a high BSA, so that patient's aortic risk would be minimized if based on weight. Ultimately, however, we could not understand how the aorta would know or care about body weight. So, in the article on which Demertzis and Grego ${ }^{1}$ are commenting, we eliminated body weight and BSA and based our predictive tool solely on height. We found that this simplified tool not only eliminated the need to calculate BSA (from height and weight) but also worked very well as a predictive tool. 


\begin{tabular}{ccccccccccccc}
\hline & & \multicolumn{10}{c}{ Aortic Size (cm) } \\
\cline { 2 - 11 } & & $\mathbf{3 . 5}$ & $\mathbf{4 . 0}$ & $\mathbf{4 . 5}$ & $\mathbf{5 . 0}$ & $\mathbf{5 . 5}$ & $\mathbf{6 . 0}$ & $\mathbf{6 . 5}$ & $\mathbf{7 . 0}$ & $\mathbf{7 . 5}$ & $\mathbf{8 . 0}$ \\
\hline $\begin{array}{c}\text { Height } \\
\text { (inches) }\end{array}$ & $(\mathbf{m})$ & & & & & & & & & & \\
$\mathbf{5 5}$ & $\mathbf{1 . 4 0}$ & 2.50 & 2.86 & 3.21 & 3.57 & 3.93 & 4.29 & 4.64 & 5.00 & 5.36 & 5.71 \\
$\mathbf{5 7}$ & $\mathbf{1 . 4 5}$ & 2.41 & 2.76 & 3.10 & 3.45 & 3.79 & 4.14 & 4.48 & 4.83 & 5.17 & 5.52 \\
$\mathbf{5 9}$ & $\mathbf{1 . 5 0}$ & 2.33 & 2.67 & 3.00 & 3.33 & 3.67 & 4.00 & 4.33 & 4.67 & 5.00 & 5.33 \\
$\mathbf{6 1}$ & $\mathbf{1 . 5 5}$ & 2.26 & 2.58 & 2.90 & 3.23 & 3.55 & 3.87 & 4.19 & 4.52 & 4.84 & 5.16 \\
$\mathbf{6 3}$ & $\mathbf{1 . 6 0}$ & 2.19 & 2.50 & 2.81 & 3.13 & 3.44 & 3.75 & 4.06 & 4.38 & 4.69 & 5.00 \\
$\mathbf{6 5}$ & $\mathbf{1 . 6 5}$ & 2.12 & 2.42 & 2.73 & 3.03 & 3.33 & 3.64 & 3.94 & 4.24 & 4.55 & 4.85 \\
$\mathbf{6 7}$ & $\mathbf{1 . 7 0}$ & 2.06 & 2.35 & 2.65 & 2.94 & 3.24 & 3.53 & 3.82 & 4.12 & 4.41 & 4.71 \\
$\mathbf{6 9}$ & $\mathbf{1 . 7 5}$ & 2.00 & 2.29 & 2.57 & 2.86 & 3.14 & 3.43 & 3.71 & 4.00 & 4.29 & 4.57 \\
$\mathbf{7 1}$ & $\mathbf{1 . 8 0}$ & 1.94 & 2.22 & 2.50 & 2.78 & 3.06 & 3.33 & 3.61 & 3.89 & 4.17 & 4.44 \\
$\mathbf{7 3}$ & $\mathbf{1 . 8 5}$ & 1.89 & 2.16 & 2.43 & 2.70 & 2.97 & 3.24 & 3.51 & 3.78 & 4.05 & 4.32 \\
$\mathbf{7 5}$ & $\mathbf{1 . 9 0}$ & 1.84 & 2.11 & 2.37 & 2.63 & 2.89 & 3.16 & 3.42 & 3.68 & 3.95 & 4.21 \\
$\mathbf{7 7}$ & $\mathbf{1 . 9 5}$ & 1.79 & 2.05 & 2.31 & 2.56 & 2.82 & 3.08 & 3.33 & 3.59 & 3.85 & 4.10 \\
$\mathbf{7 9}$ & $\mathbf{2 . 0 0}$ & 1.75 & 2.00 & 2.25 & 2.50 & 2.75 & 3.00 & 3.25 & 3.50 & 3.75 & 4.00 \\
$\mathbf{8 1}$ & $\mathbf{2 . 0 5}$ & 1.71 & 1.95 & 2.20 & 2.44 & 2.68 & 2.93 & 3.17 & 3.41 & 3.66 & 3.90 \\
\hline
\end{tabular}

Light green area indicates low risk, yellow area indicates moderate risk, orange area indicates high risk, and red area indicates severe risk.

FIGURE 1. Risk of complications (aortic dissection, rupture, and death) in ascending aortic aneurysm patients as a function of aortic diameter (horizontal axis) and height (vertical axis), with the aortic height index given within each cell. (Reproduced with permission from Zafar and colleagues. ${ }^{2}$ )

\section{Time for a Left-ward Shift in Ascending Aortic Guidelines?}

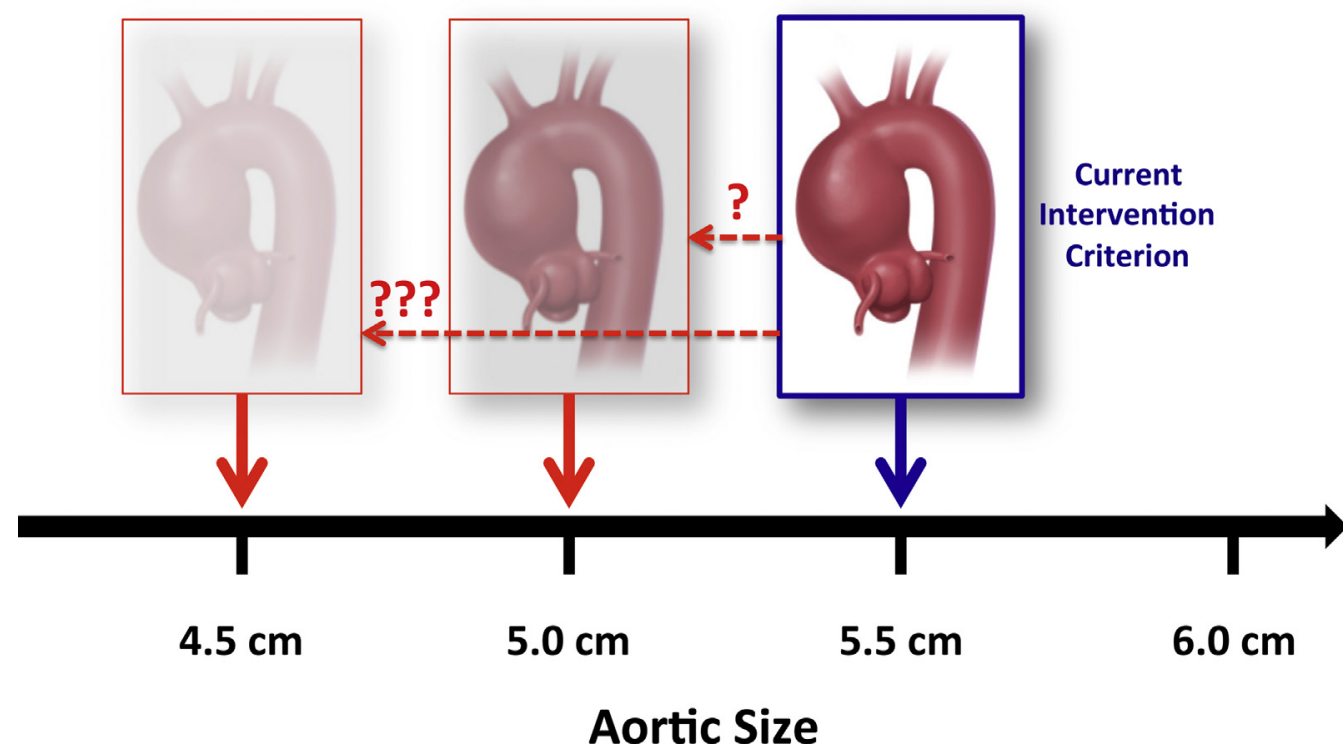

FIGURE 2. Is it time for a leftward shift in the intervention criteria for ascending aortic aneurysm? (Reproduced with permission from Ziganshin and colleagues. ${ }^{3}$ ) 
Authors have nothing to disclose with regard to commercial support.

We are very pleased to see the epidemiologic analysis presented in the letter of Demertzis and Grego. ${ }^{1}$ They calculate that, in clinical experience at their own institution (108 patients presenting with aortic dissection in the course of 17 years among a population of 350,000 inhabitants), retrospective application of our new, height-alone predictive tool would have protected a full $91 \%$ of the patients from aortic dissection. This provides reassuring "real-world" confirmation of the predictive capability of our tabular nomogram (Figure 1).

We agree fully as well with the observation of Demertzis and $\mathrm{Grego}^{1}$ that multiple evolving factors argue for earlier application of preventive aortic surgery to prevent rupture and obviate the late sequalae of aortic dissection. We have focused on specific reasons to recommend a "left shift" to earlier intervention sizes in a recent article in the Journal (Figure 2). ${ }^{3}$ These factors include (1) earlier "hinge points" in the aortic dissection curves, (2) immediate increase in aortic size at the moment of aortic dissection, (3) "centerline" method underestimation of true aortic size, (4) high safety of aortic surgery in the current era, (5) whole exome sequencing identification of especially malignant mutations, and (6) recognition that a previous dissection in the family presages that another dissection is likely to occur in other family members.

We have multiple additional predictive enhancements in evolution at our Aortic Institute. We therefore agree fully with the point made by Demertzis and Grego ${ }^{1}$ that prediction in aortic surgery is evolving favorably, although we believe that the advent of a perfect "dream come true" predictive tool still remains a reverie.

Mohammad A. Zafar, MD

Bulat A. Ziganshin, $M D, P h D$ John A. Elefteriades, $M D, P h D$ (hon) Aortic Institute at Yale-New Haven Hospital Yale University School of Medicine

New Haven, Conn

\section{References}

1. Demertzis S, Grego S. Predictability of acute aortic dissection: a dream come true? J Thorac Cardiovasc Surg. 2019;157:e324.

2. Zafar MA, Li Y, Rizzo JA, Charilaou P, Saeyeldin A, Velasquez CA, et al. Height alone, rather than body surface area, suffices for risk estimation in ascending aortic aneurysm. J Thorac Cardiovasc Surg. 2018;155:1938-50.

3. Ziganshin BA, Zafar MA, Elefteriades JA. Descending threshold for ascending aneurysmectomy: is it time for a "left-shift" in guidelines? J Thorac Cardiovasc Surg. 2019;157:37-42.

https://doi.org/10.1016/j.jtcvs.2019.01.097 